\title{
Sclerotinia Rot of Aralia elata Caused by Sclerotinia nivalis in Korea
}

\author{
Chong-Kyu Lee ${ }^{1}$, Sang-Hyun Lee ${ }^{2}$, Young-Joon Choi ${ }^{3}$, Mi-Jeong Park ${ }^{3}$ and Hyeon-Dong Shin ${ }^{3 *}$ \\ ${ }^{\prime}$ Department of Forest Resources, Jinju National University, Jinju 660-758, Korea \\ ${ }^{2}$ Division of Forest Diseases and Insect Pests, Korea Forest Research Institute, Seoul 130-712, Korea \\ ${ }^{3}$ Division of Environmental Science and Ecological Engineering, Korea University, Seoul 136-701, Korea \\ (Received on August 18, 2010; Accepted on August 30, 2010)
}

Aralia elata (Miq.) Seem., called Japanese angelica tree, is a deciduous shrub belonging to the Araliaceae, native to Russian Far East, China, Korea, and Japan. The young shoots are eaten as vegetables for various dishes in Korea, especially in spring. As the demand of this vegetable is increasing, cultivation of the tree is becoming popular. Several diseases including Phytophthora blight have been known on this plant (Lee et al., 2008). Root and rhizome rot and its damage have been noticed from several commercial fields of this crop since 1998 (Fig. 1A-C). The causal fungus of the disease was tentatively recorded as Sclerotinia sclerotiorum without data (Lee et al., 2008). To reveal the identity of the fungus, nine isolates were obtained from rotting rhizomes in Gapyeong, Jinju and Chuncheon. All isolates were typical of the genus Sclerotinia in the absence of conidia formation and rich production of sclerotia on potato dextrose agar plates (Fig. 1 D\&E). Representative three isolates were deposited in KACC (accession nos. KACC45150, 45151 and 45152) and used for further studies.

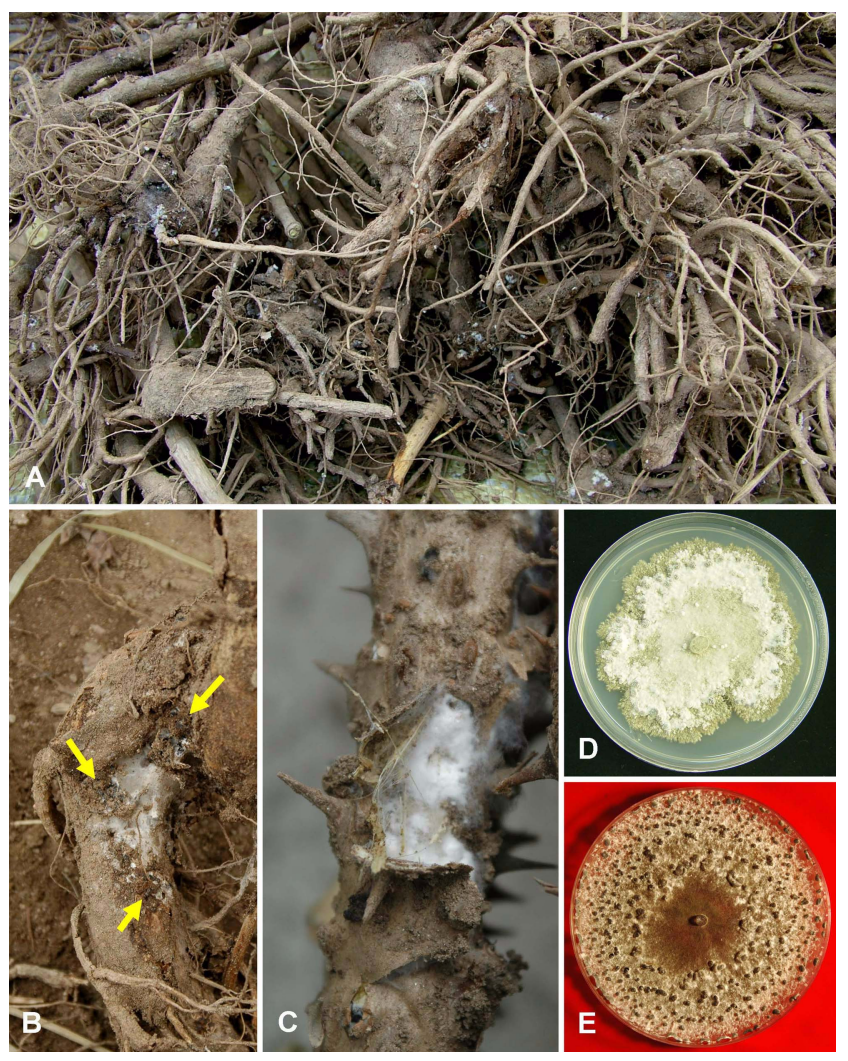

Fig. 1. (A) Young cuttings of A. elata discarded outside the greenhouse, due to heavy infestation of Sclerotinia rot. Symptoms of Sclerotinia rot on the roots (B) and stem (C) of A. elata infected with $S$. nivalis. Note the sclerotia produced on the margin of the lesion (arrows). Growth of $S$. nivalis on potato dextrose agar after 7 days of incubation (D) and formation of sclerotia after 28 days of incubation (E) at room temperature.
For pathogenicity test, ten rooted cuttings were inoculated by placing mycelial mats into the holes wounded with a cork borer $(5$ mm diam.) and then placed $3 \mathrm{~cm}$ beneath the surface of the sterilized soil. Five cuttings received sterile water for control. After two weeks of incubation, white fungus of Sclerotinia was recovered from all inoculated plants, but not from control plants, which remained asymptomatic.

Sclerotia were harvested from two-month-old cultures and used for DNA extraction. The complete ITS regions of rDNA were amplified with primers ITS5 and ITS4, and directly sequenced. The sequences obtained were identical to each other and deposited in GenBank with accession numbers of HM746662, 746663 and 746664. Phylogenetic analysis was performed using MEGA4 with neighbor-joining method (using Tajima-Nei distances). The Korean isolates shared $100 \%$ similarity with a sequence (AB516670) of $S$. nivalis I. Saito found on Arctium lappa from Japan (Fig. 2).

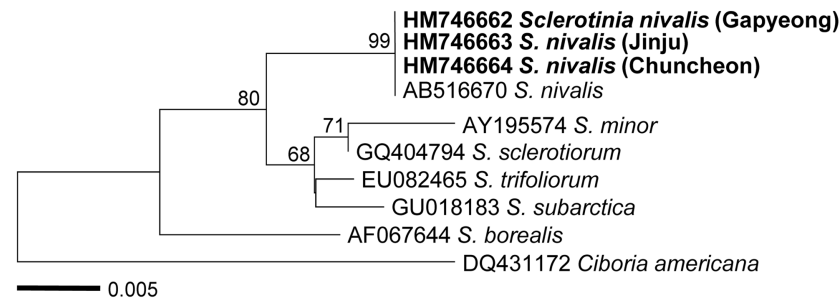

Fig. 2. Phylogenetic relationship between Sclerotinia nivalis on Aralia elata and other Sclerotinia species, inferred by neighbor-joining method using the ITS rDNA region. Numbers above the branches represent the bootstrap values. Bar=Number of nucleotide substitutions per site.

This is the first confirmed report that Japanese angelica tree is a new host for S. nivalis in Korea. This fungus is considered to have a wide host range (Li et al., 2000; Saito, 1997). It could be the most important pathogen of Japanese angelica tree, especially in nursery plots, in Korea, where the cuttings are densely planted and continuous cultivation is common.

\section{Acknowledgements}

This work was supported by the Korea Research Foundation Grant funded by Korean Government (MOEHRD, Basic Research Promotion Fund) (KRF-2008-331-F00023).

\section{References}

Lee, S. H., Kim, K. H., Seo, S. T., Moon, H. G., Shin, H. D. and Lee, C. K. 2008. Diseases of Japanese Angelica Tree and Their Control. Research Report 08-10. Korea Forest Research Institute. 57 pp. (in Korean)

Li, G. Q., Wang, D. B., Jiang, D. H., Huang, H. C. and Laroche, A. 2000. First report of Sclerotinia nivalis on lettuce in central China. Mycol. Res. 104:232-237.

Saito, I. 1997. Sclerotinia nivalis, sp. nov., the pathogen of snow mold of herbaceous dicots in northern Japan. Mycoscience 38:227-236.

*Corresponding author (hdshin@korea.ac.kr) 